\title{
Proper holomorphic curves attached to domains
}

\author{
Barbara Drinovec Drnovšek \& Marko Slapar
}

\begin{abstract}
Let $D \Subset \mathbb{C}^{n}$ be a domain with smooth boundary, of finite 1-type at a point $p \in b D$ and such that $\bar{D}$ has a basis of Stein Runge neighborhoods. Assume that there exists an analytic disc which intersects $\bar{D}$ exactly at $p$. We construct proper holomorphic maps from any open Riemann surface $S$ to $\mathbb{C}^{n}$ which are attached to $\bar{D}$ exactly at $p$.
\end{abstract}

Keywords weakly pseudoconvex domain, holomorphic curve.

MSC (2010) Primary: 32C25; Secondary: 32H02, 32H35.

\section{Introduction}

We study sufficient conditions for the existence of a proper holomorphic curve attached to the boundary of a domain, which is parametrized by an open Riemann surface or by a finite bordered Riemann surface, i.e., a one dimensional complex manifold with compact closure $\bar{S}=S \cup b S$ whose boundary $b S$ consists of finitely many closed Jordan curves.

We will denote by $\mathbb{D}$ the open unit disc in $\mathbb{C}$. Let $D \subset \mathbb{C}^{n}$ be a bounded domain with smooth boundary, let $p \in b D$ and let $\rho$ be a smooth defining function for $b D$ near $p$. Assume that $f: \mathbb{D} \rightarrow \mathbb{C}^{n}$ is a holomorphic map such that $f(0)=p$. If $\rho \circ f$ has a zero of finite order at 0 and $f(r \mathbb{D} \backslash\{0\}) \cap \bar{D}=\emptyset$ for some $r>0$ then we say that $f$ has a finite order of contact with $\bar{D}$ at $p$. The definition does not depend on the choice of the defining function. It is local and it extends to holomorphic maps from Riemann surfaces.

Theorem 1.1. Let $D \subset \mathbb{C}^{n}$ be a bounded domain with smooth boundary, $p \in b D$, and assume that $\bar{D}$ has a basis of Stein Runge neighborhoods.

(1) Given a bordered Riemann surface $S, s \in S$, and a continuous map $f: \bar{S} \rightarrow \mathbb{C}^{n}$, holomorphic on $S$, with $f(s)=p$, having a finite order of contact with $\bar{D}$ at $p$, and $f(S \backslash\{s\}) \cap \bar{D}=\emptyset$, there exists a proper holomorphic map $g: S \rightarrow \mathbb{C}^{n}$ with $g(s)=p$ and $g(S \backslash\{s\}) \cap \bar{D}=\emptyset$.

(2) Given an open Riemann surface $S$, a compact $\mathcal{O}(S)$-convex subset $K \subset S$ with the nonempty interior $\stackrel{\circ}{K}, s \in \stackrel{\circ}{K}$ and a holomorphic map $f: K \rightarrow$ $\mathbb{C}^{n}$ with $f(s)=p$, having a finite order of contact with $\bar{D}$ at $p$, and $f(K \backslash\{s\}) \cap \bar{D}=\emptyset$, there exists a proper holomorphic map $g: S \rightarrow \mathbb{C}^{n}$ with $g(s)=p$ and $g(S \backslash\{s\}) \cap \bar{D}=\emptyset$.

In particular, if $D$ is totally pseudoconvex and of finite 1-type at a point $p \in b D$, then there exist a proper holomorphic map $g: \mathbb{D} \rightarrow \mathbb{C}^{n}$ with $g(0)=p$ and $g(\mathbb{D} \backslash\{0\}) \cap \bar{D}=\emptyset$ and a proper holomorphic map $g: \mathbb{C} \rightarrow \mathbb{C}^{n}$ with $g(0)=p$ and $g(\mathbb{C} \backslash\{0\}) \cap \bar{D}=\emptyset$. 
It follows by Remmert's proper mapping theorem that the image $g(S)$ is an analytic subvariety of $\mathbb{C}^{n}$, in particular, if $n=2$, it is a global support hypersurface for $D$ at $p$ : We call an analytic hypersurface $M$ a local support hypersurface for $D$ at $p$ if there is a neighborhood $U$ of $p$ in $\mathbb{C}^{n}$ such that $M \cap U \cap \bar{D}=\{p\}$. If $M \cap \bar{D}=\{p\}$ we call $M$ a global support hypersurface. The domain $D$ is called totally pseudoconvex at $p \in b D$ if there is a local support hypersurface for $D$ at $p$ [4, 19]. Let $\rho$ be a smooth defining function for $b D$, and let $p \in b D$. If the maximum order of vanishing of $\rho \circ f$ for all one dimensional complex curves $f: \mathbb{D} \rightarrow \mathbb{C}^{n}$, with $f(0)=p$ is finite, then we say that $b D$ is of finite 1-type at $p$ [6]. Denote by $\mathcal{O}(X)$ the algebra of all holomorphic functions on the complex manifold $X$, endowed with the compact-open topology. A compact set $K$ in $X$ is said to be $\mathcal{O}(X)$-convex if for every point $x \in X \backslash K$ there exists $F \in \mathcal{O}(X)$ with $|F(x)|>\sup _{K}|F|$.

We shall actually prove part (1) in Theorem 1 .1 for domains $D$ in $(n-1)$ convex complex manifolds $X$, see Theorem 3.1, and part (2) in Theorem 1.1 for domains $D$ in Stein manifolds $X$ with the density property, see Theorem 4.1. Furthermore, the map $g$ can be chosen an immersion, and if $\operatorname{dim} X \geq 3$ then $g$ can be chosen an embedding. Moreover, we are able to attach proper holomorphic curves to more general compact sets than smoothly bounded domains $\bar{D}$; here we assume that the domain $D$ has $\mathcal{C}^{\infty}$ smooth boundary.

We call the image of a proper holomorphic map from an open Riemann surface a proper holomorphic curve. Proper holomorphic discs in pseudoconvex domains through any given point were constructed in [11] by Forstnerič and Globevnik. The results evolved in various directions, for a thorough survey of recent results we refer to [8].

We say that a domain $D \subset \mathbb{C}^{n}$ is locally convexifiable at a point $p \in b D$ if there exists a biholomorphic change of coordinates near $p$ such that in the new coordinates near $p$ the boundary $b D$ is geometrically strictly convex with respect to the side on which $D$ lies. In this case, there exists a local support hypersurface to $b D$ at $p$, in particular, $D$ is totally pseudoconvex at $p$. If $D \subset \mathbb{C}^{n}$ is a smoothly bounded strictly pseudoconvex domain then it is locally convexifiable at any boundary point by Narasimhan's lemma.

Kohn-Nirenberg example [16] shows that local support hypersurfaces do not exist in general at weakly pseudoconvex points of pseudoconvex domains. Recently, Diederich, Fornæss and Wold [7] proved that for any bounded domain $D \subset \mathbb{C}^{n}$ which is locally convexifiable and of finite 1-type near $p \in b D$, and such that $\bar{D}$ has a basis of Stein Runge neighborhoods, there exists an automorphism $\Psi$ of $\mathbb{C}^{n}$ such that $\Psi(p)$ is a global extreme point, i.e., $\Psi(\bar{D}) \cap b \mathbb{B}^{n}=\Psi(p)$, where $\mathbb{B}^{n}$ denotes the open unit ball in $\mathbb{C}^{n}$. In particular, there exists a smooth support hypersurface for $D$ at $p$.

Kolár [17, 18] constructed examples of smoothly bounded nonconvexifiable pseudoconvex domains with convex models, in particular, with local support hypersurfaces. We provide an example of a bounded pseudoconvex domain $D \subset \mathbb{C}^{2}$ with smooth boundary, such that $\bar{D}$ has a Stein Runge neighborhood basis and a 
weakly pseudoconvex point $p \in b D$ of finite 1-type such that the domain $D$ is not locally convexifiable at $p$ and that $D$ has a global support hypersurface at $p$, see Section 5 .

\section{Control of the placement of the curve near a given point}

We will consider holomorphic curves attached to more general compact sets than closures of smoothly bounded domains:

Definition 2.1. Let $L \subset \mathbb{C}^{n}$ be a compact set, $p \in L$ and $f: \mathbb{D} \rightarrow \mathbb{C}^{n}$ a holomorphic map such that $f(0)=p$. The map $f$ has a finite order of contact with $L$ at $p$ if there are $C>0, r>0$ and $k \in \mathbb{N}$ such that $\operatorname{dist}(f(z), L) \geq C|z|^{k}$ for all $|z| \leq r$. Since the definition of a finite order of contact is local it extends to maps from Riemann surfaces to complex manifolds equipped with a Riemannian metric.

For a smoothly bounded domain $D, p \in b D$ and $f: \mathbb{D} \rightarrow \mathbb{C}^{n}$ a holomorphic map such that $f(0)=p$ the definition of a finite order of contact of $f$ with $\bar{D}$ at $p$ coincides with the definition at the beginning of this note: Since the signed distance $\rho$ is a defining function for $b D$, the estimate in Definition 2.1 implies that $\rho \circ f$ has a zero of finite order at 0 . Conversely, choose a defining function $\rho$ of $b D$ near $p$. If $f: \mathbb{D} \rightarrow \mathbb{C}^{n}$ is a holomorphic map with $f(0)=p$ having a finite order of contact with $\bar{D}$ according to the definition in the Introduction, then $\rho \circ f$ has a zero of finite order at 0 and $f(r \mathbb{D} \backslash\{0\}) \cap \bar{D}=\emptyset$ for some $r>0$. Therefore, it holds that $(\rho \circ f)(z)=P_{k}(z, \bar{z})+o\left(|z|^{k}\right)$, where $P_{k}$ is a real homogeneous polynomial of degree $k$, and $P_{k}(z, \bar{z})>0$ for $z \neq 0$. This implies that there are $C>0$ and $r^{\prime}>0$ small enough such that $\operatorname{dist}(f(z), \bar{D}) \geq C|z|^{k}$ for all $|z| \leq r^{\prime}$. Thus $f$ has a finite order of contact with $\bar{D}$ at $p$ according to Definition 2.1.

If the map $f$ has a finite order of contact with compact set $L$ at $p$, then the curve that intersects $L$ only in $p$ allows small perturbations, which, in a neighborhood of $p$, intersect $L$ only in $p$. The following lemma provides appropriate small perturbations of a holomorphic disc in a neighborhood of $p$.

Lemma 2.2. Let $L \subset \mathbb{C}^{n}$ be a compact subset, $p \in L$, and $f: \mathbb{D} \rightarrow \mathbb{C}^{n} a$ holomorphic map with $f(0)=p$ and having a finite order of contact with $L$ at p. Then there exist $r, 0<r<1$, and an integer $k \geq 0$ such that for any $r^{\prime} \in(r, 1)$ there exist $\epsilon>0$ such that for any holomorphic map $g: \mathbb{D} \rightarrow \mathbb{C}^{n}$ satisfying $f^{(m)}(0)=g^{(m)}(0)$ for $m \in\{0, \ldots, k-1\}$ and $|f(z)-g(z)|<\epsilon$ for $|z| \leq r^{\prime}$ it holds that $g(r \overline{\mathbb{D}} \backslash\{0\}) \cap L=\emptyset$.

Proof. There are $C \in \mathbb{R}, r>0$ and $k \in \mathbb{N}$ such that $\operatorname{dist}(f(z), L) \geq C|z|^{k}$ for all $|z| \leq r$. Therefore, for any holomorphic map $g: \mathbb{D} \rightarrow \mathbb{C}^{n}$ satisfying $|f(z)-g(z)| \leq \frac{C}{2}|z|^{k}$ for $|z| \leq r$ it holds that $g(r \overline{\mathbb{D}} \backslash\{0\}) \cap L=\emptyset$.

Choose any $r^{\prime} \in(r, 1)$. Assume that $g: \mathbb{D} \rightarrow \mathbb{C}^{n}$ is a holomorphic map such that $f^{(m)}(0)=g^{(m)}(0)$ for $m \in\{0, \ldots, k-1\}$. By the Taylor expansion of $f-g$ 
around 0 with the estimate of the remainder and by Cauchy formula we get

$$
|f(z)-g(z)| \leq C_{1}|z|^{k} \max \left\{|f(z)-g(z)|: z \in r^{\prime} \overline{\mathbb{D}}\right\} \text { for } z \in r \overline{\mathbb{D}}
$$

where the constant $C_{1}>0$ depends only on $r$ and $r^{\prime}$. We let $\epsilon=\frac{C}{2 C_{1}}$. This proves the lemma.

\section{Proper holomorphic curves in $(n-1)$-convex complex manifolds}

Part (1) in Theorem 1.1 follows from the following theorem:

Theorem 3.1. Let $X$ be a complex manifold of $\operatorname{dim} X=n>1$ equipped with a Riemannian metric $d$ and let $L \subset X$ be a compact set. Assume that for any neighborhood $W$ of $L$ there exists a smooth exhaustion function $\rho: X \rightarrow \mathbb{R}$ that is $(n-1)$-convex on $X_{c}=\{x \in X: \rho(x)>c\}$ for some $c \in \mathbb{R}$ such that $L \subset X \backslash \bar{X}_{c} \subset W$.

Given $\epsilon>0$, a bordered Riemann surface $S, s \in S$, a compact subset $K \subset S$, a continuous map $f: \bar{S} \rightarrow X$, holomorphic on $S$ with $f(s)=p$, having a finite order of contact with $L$ at $p$ and such that $f(S \backslash\{s\}) \cap L=\emptyset$, there exists a proper holomorphic map $g: S \rightarrow X$ with $g(s)=p, g(S \backslash\{s\}) \cap L=\emptyset$ and $d(g(z), f(z))<\epsilon$ for $z \in K$.

Recall that a smooth function $\rho: X \rightarrow \mathbb{R}$ on a complex manifold of $\operatorname{dim} X=$ $n>1$ is said to be $q$-convex on an open subset $U \subset X$ (in the sense of AndreottiGrauert [1], [14, def. 1.4, p. 263]) if its Levi form $i \partial \bar{\partial} \rho$ has at most $q-1$ negative or zero eigenvalues at each point of $U$. Note that 1-convex functions are exactly strongly plurisubharmonic functions. The manifold $X$ is $q$-complete, resp. $q$ convex, if it admits a smooth exhaustion function $\rho: X \rightarrow \mathbb{R}$ which is $q$-convex on $X$, resp. on $\{x \in X: \rho(x)>c\}$ for some $c \in \mathbb{R}$. A 1-complete complex manifold is just a Stein manifold. If $X$ is a $(n-1)$-complete complex manifold with $(n-1)$-convex exhaustion function $\rho$, then we can take a sublevel set of $\rho$ for $L$ in the theorem.

The construction of the proper holomorphic map in Theorem 3.1 is inductive. The main addition to the previous constructions of proper holomorphic maps in [11, 13, 8] is the control of the placement of the disc near the point $p$ provided by Lemma 2.2. Outside this neighborhood we control the placement of the curve in such a way that no intersection with $L$ occur.

The following lemma will provide the main step in the inductive construction of a proper holomorphic map. It is a consequence of [9, Lemma 4.2] that assures that any map $f$ bellow is a core map of a spray of maps and [8, Lemma 6.3] that gives the new map $g$. Main methods in the proof are the solution to a certain Riemann-Hilbert problem which gives appropriate local corrections and the gluing of holomorphic sprays. 
Lemma 3.2. Let $X$ be a complex manifold of dimension $n>1$ equipped with a Riemannian metric $d$ and let $\rho: X \rightarrow \mathbb{R}$ be a smooth exhaustion function which is $(n-1)$-convex on $\left\{x \in X: \rho(x)>M_{1}\right\}$ for some $M_{1} \in \mathbb{R}$. Let $S$ be a bordered Riemann surface, $s \in S$, let $U \Subset S$ be an open subset, let $f: \bar{S} \rightarrow X$ be a continuous map, holomorphic on $S$, and $M_{1}<M_{2}$, such that $f(z) \in\left\{x \in X: \rho(x) \in\left(M_{1}, M_{2}\right)\right\}$ for all $z \in \bar{S} \backslash U$. Given $\varepsilon>0, M_{3}>M_{2}$, and an integer $k \geq 1$, there exists a continuous map $g: \bar{S} \rightarrow X$, holomorphic on $S$, satisfying the following properties:

(i) $g(z) \in\left\{x \in X: \rho(x) \in\left(M_{2}, M_{3}\right)\right\}$ for $z \in b S$,

(ii) $g(z) \in\left\{x \in X: \rho(x) \in\left(M_{1}, M_{3}\right)\right\}$ for $z \in \bar{S} \backslash U$,

(iii) $d(f(z), g(z))<\varepsilon$ for $z \in \bar{U}$,

(iv) the $k-1$ jets of $f$ and $g$ at $s$ are equal.

Proof of Theorem 3.1 We choose local coordinates in a neighborhood $V$ of $p$ and an open neighborhood $U$ of $s$ in $S$ such that $U$ is biholomorphic to $\mathbb{D}$ and $f(U) \Subset V$. By Lemma 2.2 there is a neighborhood $U_{0} \Subset U, \epsilon_{1} \in(0, \epsilon)$, and an integer $k>0$ such that for any holomorphic map $g: S \rightarrow X$, which has the same $k-1$ jet at $s$ as $f$, and $d(f(z), g(z))<\epsilon_{1}$ for $z \in \bar{U}$ it holds that $g(\bar{U}) \subset V$ and $g\left(\bar{U}_{0}\right) \cap L=\{p\}$.

Let $f_{0}=f$. There exist an exhaustion function $\rho: X \rightarrow \mathbb{R}$ and $M_{0} \in \mathbb{R}$ such that $\rho$ is $(n-1)$-convex on $\left\{x \in X: \rho(x)>M_{0}\right\}$, and

$$
\max _{x \in \bar{D}} \rho(x)<M_{0}<\rho\left(f_{0}(z)\right) \text { for } z \in \bar{S} \backslash U_{0} .
$$

Let $M_{-2}=\max _{x \in \bar{D}} \rho(x)$. Choose an increasing sequence $\left\{M_{i}\right\}_{i \geq-2}$ converging to $\infty$ such that

$$
M_{-2}<M_{-1}<M_{0}<\rho\left(f_{0}(z)\right)<M_{1} \text { for } z \in \bar{S} \backslash U_{0} .
$$

There is a decreasing sequence $\left\{\epsilon_{i}\right\}_{i \geq 1}$ converging to 0 such that for $i \geq-2$,

$$
\rho(x) \in\left[M_{i+1}, M_{i+3}\right] \text { and } d(x, y)<\epsilon_{i+3} \text { then } \rho(x)>M_{i} .
$$

Choose a sequence of compact subsets $\left\{K_{i}\right\}_{i \geq 1}$ in $S$ such that $\cup_{i} K_{i}=S$. We shall inductively construct a sequence of continuous maps $\left\{f_{i}: \bar{S} \rightarrow X\right\}_{i>0}$, holomorphic on $S$, and a sequence of open sets $\left\{U_{i}\right\}_{i \geq 0}, U_{i} \Subset U_{i+1}, U_{0} \cup K \subset U_{1}$, $\cup_{i} U_{i}=S$, such that the following hold for all $i \in \mathbb{Z}_{+}$:

(i) $\rho\left(f_{i}(z)\right) \in\left(M_{i}, M_{i+1}\right)$ for $z \in b S$,

(ii) $\rho\left(f_{i}(z)\right) \in\left(M_{i}, M_{i+1}\right)$ for $z \in \bar{S} \backslash U_{i+1}$,

(iii) $\rho\left(f_{i}(z)\right) \in\left(M_{i-1}, M_{i+1}\right)$ for $z \in \bar{S} \backslash U_{i}$,

(iv) $d\left(f_{i+1}(z), f_{i}(z)\right)<\frac{\epsilon_{i+1}}{2^{i+1}}$ for $z \in \bar{U}_{i+1}$,

(v) the $k-1$ jets of $f_{i+1}$ and $f_{i}$ at $s$ are equal.

Notice that by (3.1) the map $f_{0}$ meets conditions (i) and (iii) for $i=0$. Choose $U_{1}, K \cup U \Subset U_{1}$, such that (ii) for $i=0$ is satisfied. Let $j \in \mathbb{N}$ and assume that we have already constructed $f_{0}, \ldots, f_{j-1}$ and $U_{0}, \ldots, U_{j}$ that satisfy properties (i), 
(ii) and (iii) for $i=0, \ldots j-1$, and properties (iv) and (v) for $i=0, \ldots j-2$. We use Lemma 3.2 to obtain a continuous map $f_{j}: \bar{S} \rightarrow X$, holomorphic on $S$, which satisfies properties (i) and (iii) for $i=j$, and properties (iv) and (v) for $i=j-1$. Now we choose $U_{j+1}, U_{j} \cup K_{j} \Subset U_{j+1}$, such that $f_{j}$ satisfies (ii) for $i=j$. This finishes the inductive construction.

It follows by (iv) that the sequence $\left\{f_{n}\right\}$ converges uniformly on compact sets in $S$ to a holomorphic map that will be denoted by $g$. For all $j \geq-2$ and $z \in \bar{U}_{j+3}$ we get by (iv)

$$
\begin{aligned}
d\left(g(z), f_{j+2}(z)\right) & \leq d\left(f(z)_{j+2}, f_{j+3}(z)\right)+d\left(f(z)_{j+3}, f_{j+4}(z)\right)+\cdots \\
& <\frac{\epsilon_{j+3}}{2^{j+3}}+\frac{\epsilon_{j+4}}{2^{j+4}}+\cdots<\epsilon_{j+3} .
\end{aligned}
$$

The property (i) for $i=j+2$ and the property (iii) for $i=j+2$ imply that $\rho\left(f_{j+2}(z)\right) \in\left(M_{j+1}, M_{j+3}\right)$ for $z \in \bar{S} \backslash U_{j+2}$. Therefore by (3.2) and (3.3) for any $j \geq 0$ we obtain that $\rho(g(z))>M_{j}$ for $z \in \bar{U}_{j+3} \backslash U_{j+2}$, which implies that the map $g$ is proper and that $g(z) \notin L$ for $z \in S \backslash U_{0}$. Property (v) implies that the $k-1$ jets of $f$ and $g$ at $s$ are equal. By the choice of $k, U_{1}, \epsilon_{1}$ the estimate (3.3) implies that $g\left(\bar{U}_{0}\right) \cap L=\{p\}$ and that $d(g(z), f(z))<\epsilon$ for $z \in K$. This concludes the proof of the theorem.

\section{Proper holomorphic curves in manifolds with the density property}

A complex manifold $X$ enjoys the density property if the Lie algebra generated by all $\mathbb{C}$-complete holomorphic vector fields is dense in the Lie algebra of all holomorphic vector fields on $X$ (see Varolin [21, 20]). Similarly, one defines the volume density property of a complex manifold $X$ endowed with a holomorphic volume form $\omega$, by considering the Lie algebra of all holomorphic vector fields on $X$ annihilating $\omega$ (see Kaliman and Kutzschebauch [15]). Given a compact subset $K$ in a Riemann surface $S$ we say that a map $f: K \rightarrow X$ is holomorphic if it is holomorphic on some neighborhood of $K$.

Part (2) in Theorem 1.1 follows from the following theorem:

Theorem 4.1. Let $X$ be a Stein manifold of $\operatorname{dim} X=n>1$ with the density property or the volume density property equipped with a Riemannian metric $d$. Let $L \subset X$ be a $\mathcal{O}(X)$-convex compact set and $p \in L$.

Given an open Riemann surface $S$, a $\mathcal{O}(S)$-convex compact subset $K \subset S$ with nonempty interior $\stackrel{\circ}{K}, s \in \stackrel{\circ}{K}$, and a holomorphic map $f: K \rightarrow X$ with $f(s)=p$, having a finite order of contact with $L$ at $p$, and $f(K \backslash\{s\}) \cap L=\emptyset$, there exists a proper holomorphic immersion with simple double points $g: S \rightarrow X$ with $g(s)=p$, $g(S \backslash\{s\}) \cap L=\emptyset$, and $d(g(z), f(z))<\epsilon$ for $z \in K$. If $n \geq 3$ then $g$ can be chosen an embedding. 
In particular, if $L$ is a polynomially convex compact subset in $\mathbb{C}^{n}, p \in L$, and $f: \mathbb{D} \rightarrow X$ is a holomorphic map with $f(0)=p$, having a finite order of contact with $L$ at $p$, then for any open Riemann surface $S, s \in S$, there is a proper holomorphic immersion with simple double points $g: S \rightarrow X$ with $g(s)=p$, $g(S \backslash\{s\}) \cap L=\emptyset$. If $n \geq 3$ then $g$ can be chosen an embedding.

Andrist and Wold [3] proved that an open Riemann surface $S$ immerses into Stein manifold $X$ of $\operatorname{dim} X \geq 2$ with the (volume) density property properly holomorphically. In the case $\operatorname{dim} X \geq 3$ they were able to choose this immersion to be an embedding. In [2] the authors extended this result and proved that any Stein manifold $S$ can be embedded into Stein manifold $X$ with the (volume) density property properly holomorphically if $\operatorname{dim} X \geq 2 \operatorname{dim} S+1$.

Forstnerič and Ritter [12] proved that holomorphic maps from Stein manifolds $S$ of dimension $<n$ to the complement of a compact convex set $L$ in $\mathbb{C}^{n}$ satisfy the basic Oka property with approximation and interpolation. Furthermore, if $2 \operatorname{dim} S \leq n$ they proved that for any polynomially convex subset $L$ of $\mathbb{C}^{n}$ and for any holomorphic map $f$ defined on a $\mathcal{O}(S)$-convex compact set $K \subset S$ such that $f(b K) \subset \mathbb{C}^{n} \backslash L$ there exists a proper holomorphic map $g: S \rightarrow \mathbb{C}^{n}$ such that $g(S \backslash K) \subset \mathbb{C}^{n} \backslash L$. The proof depends on Andersén-Lempert-ForstneričRosay theorem on approximation of isotopies of injective holomorphic maps by holomorphic automorphisms which also holds on Stein manifolds with the (volume) density property. That enabled Forstnerič to extend the above results [10]. More precisely, in the proof of Theorem 4.1 we use [10, Remark 1.3]. The interpolation of a higher order jet can be easily built in the construction:

Theorem 4.2. Let $X$ be a Stein manifold of $\operatorname{dim} X=n>1$ with the density property or the volume density property equipped with a Riemannian metric $d$. Let $L \subset X$ be a compact $\mathcal{O}(X)$-convex set. Assume that $S$ is an open Riemann surface, $K$ is a compact $\mathcal{O}(S)$-convex set in $S$ and $s \in S$.

Let $U$ be an open neighborhood of $K$ and $f: U \cup\{s\} \rightarrow X$ a holomorphic map such that $f(b K) \cap L=\emptyset$. Given $\epsilon>0$ and an integer $k \geq 1$ there exists a proper holomorphic immersion with simple double points $g: S \rightarrow X$ such that the $k-1$ jets of $f$ and $g$ at $s$ are equal, satisfying $g(S \backslash K) \subset X \backslash L$, and $d(f(z), g(z))<\epsilon$ for $z \in K$. If $n \geq 3$ then $g$ can be chosen an embedding.

Proof of Theorem 4.1 We choose local coordinates in a neighborhood $V$ of $p$ and an open neighborhood $U^{\prime} \subset U$ of $s$ in $S$ such that $U^{\prime}$ is biholomorphic to $\mathbb{D}$ and $f\left(U^{\prime}\right) \Subset V$. By Lemma 2.2 there is a neighborhood $U^{\prime \prime} \Subset U^{\prime}, \epsilon_{1} \in(0, \epsilon)$, and an integer $k>0$ such that for any holomorphic map $g: S \rightarrow X$ such that the $k-1$ jets of $f$ and $g$ at $s$ are equal, and $d(f(z), g(z))<\epsilon_{1}$ for $z \in U^{\prime}$ it holds that $g\left(\bar{U}^{\prime \prime}\right) \subset V$ and $g\left(\bar{U}^{\prime \prime} \backslash\{s\}\right) \cap L=\emptyset$. There is $\epsilon_{2} \in\left(0, \epsilon_{1}\right)$ such that $\operatorname{dist}(f(z), L)>\epsilon_{2}$ for $z \in K \backslash U^{\prime \prime}$.

By Theorem 4.2 there exists a proper holomorphic immersion with simple double points $g: S \rightarrow X$ such that the $k-1$ jets of $f$ and $g$ at $s$ are equal, satisfying 
$g(S \backslash K) \subset X \backslash L$, and $d(f(z), g(z))<\epsilon_{2}$ for $z \in K$. The choice of $\epsilon_{2}$ implies that $g(K \backslash\{s\}) \cap L=\emptyset$. This proves the theorem.

\section{Example}

The domain

$$
D=\left\{(z, w) \in \mathbb{C}^{2}:-\Re w+\frac{1}{5}|w|^{2}+|z|^{8}+|z|^{2} \Re\left(z^{6}\right)+10|z|^{10}+|w z|^{2}<0\right\}
$$

has the following properties:

- $D$ is a bounded pseudoconvex domain in $\mathbb{C}^{2}$ with smooth boundary,

- $\bar{D}$ has a Stein Runge neighborhood basis,

- $0 \in b D$ is a weakly pseudoconvex point of finite 1-type,

- $D$ is not locally convexifiable at 0 ,

- $D$ has a global support hypersurface at 0 .

The domain $D$ is a modification of the Kohn-Nirenberg domain

$$
D^{\prime}=\left\{(z, w) \in \mathbb{C}^{2}:-\Re w+|z|^{8}+\frac{15}{7}|z|^{2} \Re\left(z^{6}\right)+|w z|^{2}<0\right\},
$$

which is weakly pseudoconvex domain with smooth boundary that does not have a support hypersurface at 0 . Calamai [5] constructed a bounded weakly pseudoconvex domain with smooth boundary that does not have a support hypersurface at 0. Kolár [17, 18] constructed examples of smoothly bounded nonconvexifiable pseudoconvex domains with local support hypersurfaces. Our example is built on these constructions.

Proof. Let

$$
\rho(z, w)=-\Re w+\frac{1}{5}|w|^{2}+|z|^{8}+|z|^{2} \Re\left(z^{6}\right)+10|z|^{10}+|w z|^{2},(z, w) \in \mathbb{C}^{2} .
$$

It is not difficult to check that the level set $\{\rho(z, w)=0\}=b D$ is smooth. To see that $D$ is bounded write $\rho(z, w)=\frac{1}{5}\left|w-\frac{5}{2}\right|^{2}-\frac{5}{4}+|z|^{8}\left(1+\frac{\Re\left(z^{6}\right)}{z^{6}}\right)+10|z|^{10}+|w z|^{2}$ and we derive that

$$
\left\{(z, w) \in \mathbb{C}^{2}: \rho(z, w)<0\right\} \subset\left\{(z, w) \in \mathbb{C}^{2}:\left|w-\frac{5}{2}\right|^{2}<\frac{5}{2},|z|<\sqrt[10]{\frac{1}{8}}\right\} .
$$

We calculate the Levi form of $\rho$

$$
\mathcal{L}_{\rho}(z, w)=\left[\begin{array}{cc}
16|z|^{6}+7 \Re\left(z^{6}\right)+250|z|^{8}+|w|^{2} & w \bar{z} \\
\bar{w} z & \frac{1}{5}+|z|^{2}
\end{array}\right] .
$$

It is easy to see that the Levi form is strictly positive definite on $\mathbb{C}^{2} \backslash\{0,0\}$. Therefore $D$ is pseudoconvex, $\bar{D}$ has a Stein Runge neighborhood basis, and 0 is a weakly pseudoconvex point.

To prove that $D$ is not locally convexifiable at 0 we compute the defining equation for $b D$ near 0 :

$$
\Re w=|z|^{8}+|z|_{8}^{2} \Re\left(z^{6}\right)+o\left(\Im w,|z|^{8}\right),
$$


and by [17, Proposition 3] the domain $D$ is not convex in any holomorphic coordinates around 0 . From this expression it also follows that 0 is a point of finite type 8 . Since $\rho(z, 0)>0$ for $z \neq 0, \mathbb{C} \times\{0\}$ is a global supporting hypersurface for $b D$ at 0 .

Acknowledgements. The authors thank Mike Lawrence for bringing the question of the existence of proper holomorphic discs attached to boundaries of pseudoconvex domains to their attention and Franc Forstnerič for helpful discussions.

The authors are supported in part by the research program P1-0291 and grants J1-5432 and J1-9104 from ARRS, Republic of Slovenia.

\section{References}

[1] A. Andreotti and H. Grauert. Théorème de finitude pour la cohomologie des espaces complexes. Bull. Soc. Math. France, 90:193-259, 1962.

[2] R. Andrist, F. Forstnerič, T. Ritter, and E. F. Wold. Proper holomorphic embeddings into Stein manifolds with the density property. J. Anal. Math., 130:135-150, 2016.

[3] R. B. Andrist and E. F. Wold. Riemann surfaces in Stein manifolds with the density property. Ann. Inst. Fourier (Grenoble), 64(2):681-697, 2014.

[4] H. Behnke and P. Thullen. Theorie der Funktionen mehrerer komplexer Veränderlichen. Ergebnisse der Mathematik und ihrer Grenzgebiete, Band 51. Zweite, erweiterte Auflage. Herausgegeben von R. Remmert. Unter Mitarbeit von W. Barth, O. Forster, H. Holmann, W. Kaup, H. Kerner, H.-J. Reiffen, G. Scheja und K. Spallek. Springer-Verlag, Berlin-New York, 1970.

[5] S. Calamai. A bounded Kohn Nirenberg domain. Bull. Korean Math. Soc., 51(5):1339-1345, 2014.

[6] J. P. D'Angelo. Several complex variables and the geometry of real hypersurfaces. Studies in Advanced Mathematics. CRC Press, Boca Raton, FL, 1993.

[7] K. Diederich, J. E. Fornæss, and E. F. Wold. Exposing points on the boundary of a strictly pseudoconvex or a locally convexifiable domain of finite 1-type. J. Geom. Anal., 24(4):21242134, 2014.

[8] B. Drinovec Drnovšek and F. Forstnerič. Holomorphic curves in complex spaces. Duke Math. J., 139(2):203-253, 2007.

[9] B. Drinovec Drnovšek and F. Forstnerič. Strongly pseudoconvex domains as subvarieties of complex manifolds. Amer. J. Math., 132(2):331-360, 2010.

[10] F. Forstnerič. Proper holomorphic immersions into stein manifolds with the density property. J. Anal. Math., in press.

[11] F. Forstnerič and J. Globevnik. Discs in pseudoconvex domains. Comment. Math. Helv., 67(1):129-145, 1992.

[12] F. Forstnerič and T. Ritter. Oka properties of ball complements. Math. Z., 277(1-2):325-338, 2014.

[13] J. Globevnik. Discs in Stein manifolds. Indiana Univ. Math. J., 49(2):553-574, 2000.

[14] H. Grauert. Theory of $q$-convexity and $q$-concavity. In Several complex variables, VII, volume 74 of Encyclopaedia Math. Sci., pages 259-284. Springer, Berlin, 1994.

[15] S. Kaliman and F. Kutzschebauch. Algebraic volume density property of affine algebraic manifolds. Invent. Math., 181(3):605-647, 2010.

[16] J. J. Kohn and L. Nirenberg. A pseudo-convex domain not admitting a holomorphic support function. Math. Ann., 201:265-268, 1973. 
[17] M. Kolár. Convexifiability and supporting functions in $\mathbf{C}^{2}$. Math. Res. Lett., 2(4):505-513, 1995.

[18] M. Kolár. Generalized models and local invariants of Kohn-Nirenberg domains. Math. Z., 259(2):277-286, 2008.

[19] R. M. Range. The Carathéodory metric and holomorphic maps on a class of weakly pseudoconvex domains. Pacific J. Math., 78(1):173-189, 1978.

[20] D. Varolin. The density property for complex manifolds and geometric structures. II. Internat. J. Math., 11(6):837-847, 2000.

[21] D. Varolin. The density property for complex manifolds and geometric structures. J. Geom. Anal., 11(1):135-160, 2001.

\section{Barbara Drinovec Drnovšek}

Faculty of Mathematics and Physics, University of Ljubljana, Jadranska 19, SI1000 Ljubljana, Slovenia, and

Institute of Mathematics, Physics and Mechanics, Jadranska 19, SI-1000 Ljubljana, Slovenia.

e-mail: barbara.drinovecefmf.uni-lj.si

\section{Marko Slapar}

Faculty of Education, University of Ljubljana, Kardeljeva ploščad 16, SI-1000 Ljubljana, Slovenia,

Faculty of Mathematics and Physics, University of Ljubljana, Jadranska 19, SI1000 Ljubljana, Slovenia, and

Institute of Mathematics, Physics and Mechanics, Jadranska 19, SI-1000 Ljubljana, Slovenia.

e-mail: marko.slapar@pef.uni-lj.si 